\title{
Serum Lactate Levels as a Predictor of Short-Term Mortality in Critically Ill Patients with Liver Cirrhosis
}

\author{
F.M.Khalil, M.A.El-Assal, A.M.Dabour, A.K.El-Alfy and M.d.Idriss \\ Internal Medicine Dept., Faculty of Medicine, Benha Univ., Benha, Egypt \\ E-Mail: Mohammed@gmail.com
}

\begin{abstract}
Intense hepatic hindrance is related with expanded lactate levels. Furthermore, in patients with fulminant hepatic disappointment and in ICU patients with cirrhosis, expanded lactate was related with helpless result The point of the this investigation was to survey the standard of serum lactate level as an indicator of momentary mortality in basically sick patients with We incorporated a sum of 70 cases determined to have constant liver illness who were isolated into three gatherings; bunch An included 30 cases with decompensated infection and hepatic encephalopathy, bunch B included 30 cases with decompensated sickness and upper GI dying, and gathering C incorporated the excess 10 cases who had decompensated liver infection without intense intricacy. All cases were exposed to finish history taking, careful actual assessment, and routine lab and radiological examinations and Initial serum lactate levels When it comes to lactate levels was altogether higher in non-survivors contrasted with survivors $(7.66$ versus $3.11 \mathrm{mmol} / \mathrm{l}-\mathrm{p}<0.001)$. It was likewise higher in gatherings An and B as they encountered higher mortality contrasted with bunch C. Utilizing a cut off estimation of $6.8 \mathrm{mmol} / \mathrm{l}$, lactate had affectability and particularity of 97.8 and $95.7 \%$ separately in foreseeing mortality in basically sick cirrhotic patients, with a precision of $95.2 \%$. Lactate can be utilized as a helpful indicator for mortality in fundamentally sick cirrhotic patients. Along with CTP and APACHE II scores, they are autonomous indicators of mortality in these cases.
\end{abstract}

Keywords: Liver Cirrhosis mortality rate, Serum Lactate.

\section{Introduction}

Lactate levels might be a helpful apparatus for evaluating seriousness of infection in basically sick patients with cirrhosis admitted to the middle of the road care units. It has been demonstrated that expanded lactate levels and diminished lactate freedom are related with mortality in fundamentally sick patients [1].

Death rates in patients with cirrhosis treated at the middle of the road care unit range somewhere in the range of $40 \%$ and $60 \%$. Diverse scoring frameworks have been created to evaluate forecast in patients with cirrhosis and ACLF. Appraisal of forecast, particularly in patients with cirrhosis at the halfway consideration unit, is of vital significance to direct restorative measures $[1,2]$.

As of late, lactate has been fused in an assessment based score for foreseeing result in fundamentally sick patients with constant liver illness, and the Asian Pacific Association for the Study of the Liver (APASL) even consolidated lactate levels in its APASL ACLF research consortium (AARC)- ACLF score [3].

Nonetheless, the unmistakable prognostic estimation of lactate levels concerning result, and the likely commitment of lactate to current scoring frameworks have not been assessed in countless fundamentally sick patients with cirrhosis. Current proposals on administration of basically sick patients with cirrhosis recommend the "rehashed estimation of blood lactate levels, despite the fact that the understanding might be convoluted by hindered freedom in cirrhosis [4].

The point of the this examination was to evaluate the standard of serum lactate level as an indicator of transient mortality in fundamentally sick patients with liver cirrhosis admitted to the transitional consideration units

\section{Patient and method}

This was a prospective descriptive study included 70 patients of both genders with proved liver cirrhosis by ultrasound and were divided into 3 groups;

- Group A: Included 30 patients with decompensated liver cirrhosis with hepatic encephalopathy.

- Group B: Included 30 patients with decompensated liver cirrhosis with upper GIT bleeding.

- Group C: Included 10 patients with decompensated liver cirrhosis without acute complication used as a control group.

\subsection{Inclusion criteria}

- Age $>18$ years.

- Patients who had an arterial blood gas analysis performed within 2 hours from Internal Medicine department admission

\subsection{Exclusion criteria}

- Missing blood gas analysis within the first 2 hours from Internal Medicine department admission

- Hypoxia

- Drugs increase level of serum lactate

- Other causes of metabolic acidosis

\subsection{All patient were subjected to}

- Glasgow Coma Scale (GCS)

- MELD score

- Sequential Organ Failure Assessment Score (SOFA.

- Child-Pugh Score.

- Complete blood count.

- Blood urea (mg/dl), serum creatinine $(\mathrm{mg} / \mathrm{dl})$, serum sodium $(\mathrm{mg} / \mathrm{dl})$, and serum potassium $(\mathrm{mg} / \mathrm{dl})$. 
- Liver function tests (S. Albumin, S. Bilirubin, AST, ALT and INR),

- Serum albumin and random blood glucose $(\mathrm{mg} / \mathrm{dl})$

- Initial serum lactate levels.

\subsection{Outcomes were estimated by}

- Mortality.

- Hospital length of stay.

- ICU length of stay.

\section{Results}

The segment information of the cases inside the distinctive investigation bunches is shown in table (2). The mean age of the cases demonstrated no genuinely critical distinction between the investigation gatherings $(\mathrm{P}=0.192)$, the mean age of the cases in gathering $\mathrm{A}, \mathrm{B}$ and $C$ was $65.43 \pm 7.6$ years, $66.17 \pm 6.8$ years and 64.92 \pm 6.1 years individually. The sex dissemination uncovered no measurably huge contrast between the investigation gatherings $(\mathrm{p}=0.146)$. most of the cases in the three investigation bunches were guys $(63.3 \%, 73.3 \%$ and $70 \%$ separately).

The middle span of cirrhosis in gathering A was 4 years (range 2 to 9 years), in gathering B was 5 years (range 1 to 8 years) and in gathering $C$ was 4 years (range 1 to 8 years) with no genuinely huge contrast between the three gatherings $(\mathrm{p}=0.419)$. Past hospitalization was accounted for in $30 \%$ in gathering $\mathrm{A}$, $23.33 \%$ in gathering B and $20 \%$ in gathering $\mathrm{C}$ with no genuinely critical contrast between the investigation gatherings.

As respect research facility biochemical boundaries, there was a critical distinction between the three gatherings with respect to RBCs, BUN, Urea, creatinine, and egg whites, while there was no huge contrast as respect platelets, WBCs, and liver proteins.

The mean serum lactate level in gathering A was 6.94 $\pm 0.88 \mathrm{mmol} / \mathrm{l}$, in gathering $\mathrm{B}$ was $6.23 \pm 0.76 \mathrm{mmol} / \mathrm{l}$ and $3.15 \pm 0.32 \mathrm{mmol} / \mathrm{l}$ in gathering $\mathrm{C}$ with genuinely critical distinction between the investigation gatherings $(p=0.005)$. the serum lactate level was genuinely critical higher in gathering An and bunch B as contrasted and gathering $\mathrm{C}$, be that as it may, there was no measurably huge distinction between bunch An and bunch B.

The rate of mortality was $36.67 \%$ in gathering $\mathrm{A}$, $40 \%$ in gathering B and $20 \%$ in gathering $\mathrm{C}$ with genuinely huge distinction between the examination gatherings $(\mathrm{p}=0.039)$. the frequency of mortality was higher in gathering An and bunch B was altogether higher as contrasted and gathering while there was no genuinely huge distinction between bunch An and bunch
B. the general rate of mortality was $(35.71 \%), 25$ cases from 70 cases.

The mean age of the cases in the non-survivor bunch was $69.73 \pm 8.67$ years and in the survivor bunch was $61.13 \pm 9.61$ years with genuinely critical contrast between the two gatherings. There were 18 guys $(58.1 \%)$ and 13 females (41.9\%) in the non-survivor gathering and $25(64.1 \%)$ guys and $14(35.9 \%)$ females in the survivor bunch with no genuinely huge distinction between the two gatherings. The BMI didn't uncover any critical distinction between the two gatherings.

By contrasting the various things of the underlying clinical assessment inside the two gatherings, the beat, MAP, RR and temperature didn't uncover any critical distinction between the two gatherings, anyway the middle GCS in the survivor bunch was fundamentally higher than the non-survivor gathering $(\mathrm{p}=0.016)$.

Among the tried lab boundaries in the two investigation gatherings, just the accompanying boundaries indicated genuinely critical higher qualities in the non-survivors bunch when contrasted with the survivors bunch including WBCs, BUN, Serum urea and Serum creatinine.

All the liver capacities were fundamentally higher in the non-survivors bunches as contrasted and the survivors-bunch aside from egg whites that was measurably altogether lower in the non-survivors gathering and direct bilirubin that didn't uncover a genuinely huge distinction.

Merge score, CTP score, APACHE II score and SOFA score were essentially higher in the non-survivors bunches as contrasted and the survivors-gathering.

The mean serum lactate level in the non-survivor bunch was $7.66 \pm 0.86 \mathrm{mmol} / \mathrm{l}$ that was essentially higher as contrasted and the survivor gathering $(3.11 \pm 0.57$ $\mathrm{mmol} / \mathrm{l})(\mathrm{p}<0.001)$.

the length of medical clinic remain and length of ICU stay were measurably critical longer in the non-survivors $(\mathrm{p}<0.001)$.

The cutoff purpose of lactate at admission to separate between non-survivors from survivors was $>6.81$ with $97.8 \%$ affectability and $95.7 \%$ explicitness.

with univariate relapse investigation age, GCS, MELD score, CTB score, APACHE II score and Lactate level were demonstrated to be hazard factors for mortality in fundamentally sick cirrhotic patients. Nonetheless, with multivariate relapse investigation, CTB score, APACHE II score and lactate level were appeared as free indicators for mortality with the most noteworthy centrality with lactate level

Table (1) Univariate and multivariate analysis of predictors of mortality in critically ill cirrhotic patients.

\begin{tabular}{lcccc}
\hline Variables & Univariate & \multicolumn{3}{c}{ Multivariate analysis } \\
\cline { 3 - 5 } & analysis & OR & 95\% CI for OR & P value \\
\hline Age & $0.012^{*}$ & 0.932 & $0.618-1.237$ & 0.126 \\
Male gender & 0.215 & & & \\
BMI & 0.467 & & & \\
\hline
\end{tabular}




\begin{tabular}{lcccc}
\hline Table (1) Continue & & & & \\
\hline GCS & $0.004^{*}$ & 1.029 & $0.811-1.498$ & 0.063 \\
MELD score & $0.011^{*}$ & 0.462 & $0.314-0.869$ & 0.109 \\
CTP score & $<0.001^{*}$ & 1.824 & $1.273-2.982$ & $0.043^{*}$ \\
APACHE II score & $0.036^{*}$ & 20.317 & $2.211-2.764$ & $0.031^{*}$ \\
SOFA score & 0.246 & & & \\
Lactate level & $<0.001^{*}$ & 3.263 & $2.187-4.293$ & $0.001^{*}$ \\
\hline
\end{tabular}

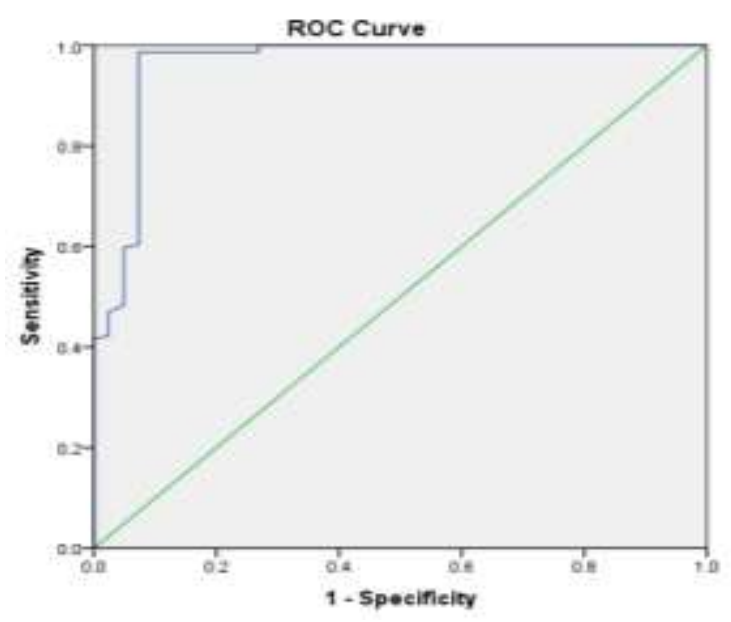

Fig (1) ROC curve of lactate in predicting survival.

\section{Discussion}

As of late, lactate has been joined in an assessment based score for foreseeing result in basically sick patients with ongoing liver infection [5], and the Asian Pacific Association for the Study of the Liver (APASL) even consolidated lactate levels in its AARC-ACLF score [3].

Be that as it may, the unmistakable prognostic estimation of lactate-levels and lactate-freedom concerning result, and the expected commitment of lactate to current scoring frameworks have not been assessed in countless basically sick patients with cirrhosis.

Mortality was experienced in 25 cases in the current examination $(35.71 \%)$. This is steady with the outcomes distributed by Saliba et al. who demonstrated that the ICU death pace of cirrhotic patients went from 34 to $69 \%$ [6].

Parkash et al. revealed that $47 \%$ of all patients who were admitted to the ICU with liver illness kicked the bucket [7]. Drolz and his partners revealed that 234 out of 566 cases had mortality (41.34\%) [4].

A past Egyptian investigation announced higher death rates contrasted with our own as mortality was experienced in 69 cases out of an aggregate of 120 cases $(57.5 \%)$ [8].

Likewise, in another examination, medical clinic mortality of patients with ESLD admitted to the ICU was high (>55\%) [9]. In addition, Tu et al. demonstrated that in cirrhotic patients admitted to ICUs in a tertiary consideration college medical clinic in Taiwan the general clinic mortality was $59.9 \%$ [10].

In the current investigation, there was a critical contrast between bunches $\mathrm{A}, \mathrm{B}$ and $\mathrm{C}$ with respect to the frequency of mortality. It was essentially higher in cases with intense intricacies (bunches An and B - 36.67 and $40 \%$ individually) contrasted with cases without complexities (bunch C $-20 \%$ ) ( $<<0.001)$. Studies demonstrated that patients with decompensated cirrhosis had a helpless forecast, especially when they create confusions identified with ESLD, for example, HE, SBP, or GIT seeping with or without lessened renal capacity $[11,12]$.

A few investigations announced that after the advancement of the main scene of only he the endurance likelihood is $42 \%$ at 1 year of development and $23 \%$ at 3 years $[13,14]$. In another investigation, HE involved the absolute most normal introduction of liver infection, as observed in $47 \%$ of patients, of whom half passed on in clinic (7). Besides, different examinations detailed that $\mathrm{HE}$ was the most well-known inconvenience of cirrhosis expecting admission to the ICU (33\%) where it additionally was the most well-known reason for death (half) $[15,16]$.

In our examination, we likewise ordered patients as per the essential result into survivors and non survivors. Age was fundamentally more seasoned in non-survivors (69.73 versus 61.13 years in survivors $-\mathrm{p}=0.041$ ).

Drolz et al. have revealed that age was altogether more seasoned in non-survivors (60 versus 56 years in survivor gathering $-\mathrm{p}<0.01)$. Mature age was a critical danger factor for mortality $(p=0.0155)$ [4]. This concurs with our discoveries.

With regards to lactate levels in the current investigation, it was altogether higher in non-survivors contrasted with survivors (7.66 versus $3.11 \mathrm{mmol} / \mathrm{l}-\mathrm{p}<$ 0.001). It was likewise higher in gatherings An and B as 
they encountered higher mortality contrasted with bunch C.

Past examinations exhibited that hepatic brokenness is related with raised lactate levels, autonomous from boundaries associated with intense circulatory disappointment [17].

As per our outcomes, Drolz and his partners detailed a critical distinction among survivors and non-survivors with respect to confirmation lactate levels ( 2.0 versus 3.9 $\mathrm{mmol} / \mathrm{l}$ individually $-\mathrm{p}<0.001)$. In addition, follow up lactate levels were likewise raised in a similar gathering in a similar report. Confirmation blood vessel lactate was a huge danger factor for mortality $(\mathrm{p}<0.001)$ [4].

Furthermore, another investigation revealed that lactate levels were fundamentally raised in non-survivors (7.16 versus $4.08 \mathrm{mmol} / \mathrm{l}$ in survivors $-\mathrm{p}<0.001$ ) [18].

Zhu and his partners detailed fundamentally higher estimations of lactate in non-survivors contrasted with survivors (3 versus $1.7 \mathrm{mmol} / \mathrm{l}$ separately $-\mathrm{p}<0.001$ ) ( 19). Tas et al. likewise announced that non-survivors had mean lactate level of $5.47 \mathrm{mmol} / \mathrm{l}$, while it was 1.76 $\mathrm{mmol} / \mathrm{l}$ in survivors $(\mathrm{p}<0.001)$ [20].

Real et al. led a multicenter randomized clinical preliminary of 187 patients going through early sepsis revival, and uncovered that liver brokenness was fundamentally connected with higher gauge lactate levels. Albeit hindered lactate leeway and standardization was seen in cutting edge liver infection, the outcome didn't convert into a distinction in mortality [21].

In our investigation, utilizing a cut off estimation of $6.8 \mathrm{mmol} / \mathrm{l}$, lactate had affectability and explicitness of 97.8 and $95.7 \%$ individually in foreseeing mortality in basically sick cirrhotic patients, with an exactness of $95.2 \%$.

In another examination which utilized a cut off estimation of $2 \mathrm{mmol} / \mathrm{l}$, serum lactate had affectability and particularity of 86 and $33 \%$ separately in anticipating mortality in decompensated liver cirrhosis cases (18). Zhu et al. utilized a cut off estimation of $2.6 \mathrm{mmol} / \mathrm{l}$ which had affectability and explicitness of 79.16 and $56.58 \%$ separately [19].

In the current investigation, multivariate examination uncovered that high CTP score, high APACHE II score, and high lactate levels were free indicators for mortality in fundamentally sick cirrhotic patients.

In another examination, free danger factors for inclinic mortality were age, hypoalbuminemia, INR, and the changed Sequential Organ Failure Assessment score [22]. Zhu et al. announced that mature age, hepatocellular carcinoma, low egg whites, high GGT, high creatinine, and high lactate were free indicators of mortality in fundamentally sick cirrhotic patients [19].

Tas et al. announced that high serum lactate, APACHE II score, SOFA score, MELD score, and CTP were free indicators of mortality in fundamentally sick cirrhotic patients [20]. Heuman et al. expressed that in multivariate investigation the MELD score, tireless ascites, and low $\mathrm{Na}(<130 \mathrm{mmol} / \mathrm{l})$ were the lone factors autonomously connected with half year mortality [23].
Different examinations indicated that HE, MELD, and CTP scores were the solitary variables related freely with shortterm and long haul mortality in cirrhotic patients $([13,14]$.

The distinction between these danger factors between various investigations could be because of various populace, test size, understanding rules, sickness models, and the executives choices.

Our investigation has a few impediments; most importantly, it is a solitary community study. Likewise, we incorporated a generally little example size. Also, our gathering order was principally relying upon confusions (HE and hematemesis), as opposed to mortality. Thus, more investigations including more cases from various hepatology focuses ought to be directed soon.

\section{Conclusion}

Lactate can be utilized as a helpful indicator for mortality in basically sick cirrhotic patients. Along with CTP and APACHE II scores, they are autonomous indicators of mortality in these cases.

\section{References}

[1] V.Fuhrmann, T.Whitehouse, J.Wendon. The ten tips to manage critically ill patients with acute-onchronic liver failure. Intensive care medicine, Vol.44(11), PP.1932-1935,2018.

[2] P.Huebener, M.Sterneck, K.Bangert. Stabilisation of acute-on-chronic liver failure patients before liver transplantation predicts post-transplant survival. Alimentary pharmacology \& therapeutics, Vol.47(11),pp.1502-1510,2018.

[3] A.Choudhury, A.Jindal, R.Maiwall. Liver failure determines the outcome in patients of acute-onchronic liver failure (ACLF): comparison of APASL ACLF research consortium (AARC) and CLIF-SOFA models. Hepatology international, Vol.11(5), PP.461-471,2017.

[4] A.Drolz, T.Horvatits, K.Rutter. Lactate improves prediction of short-term mortality in critically Ill patients with cirrhosis: a multinational study. Hepatology, Vol.69(1), PP.258-269,2019.

[5] C.Edmark, M.J.McPhail, M.Bell. LiFe: a liver injury score to predict outcome in critically ill patients. Intensive care medicine, Vol.42(3), PP.361$369,2016$.

[6] F.Saliba, P.Ichaï, E.Levesque. Cirrhotic patients in the ICU: prognostic markers and outcome. Current opinion in critical care, Vol.19(2), PP.154$160,2013$.

[7] O.Parkash, R.Iqbal, F.Jafri. Frequency of poor quality of life and predictors of health related quality of life in cirrhosis at a tertiary care hospital Pakistan. BMC research notes, Vol.5 (1),pp. $\leqslant \leqslant 7$ ,2012.

[8] H.M.Nafeh, S.S.Abdelmoneim, S.M.Hassany. Risk factors and outcome in ICU patients with end-stage liver disease. Journal of the Arab Society for Medical Research, Vol.9(1),pp.33, 2014. 
[9] A.J.O’Brien, C.A.Welch, M.Singer. Prevalence and outcome of cirrhosis patients admitted to UK intensive care: a comparison against dialysisdependent chronic renal failure patients. Intensive care medicine, Vol.38(6), PP.991-1000,2012.

[10]K.H.Tu, C.C.Jenq, M.H.Tsai. Outcome scoring systems for short-term prognosis in critically ill cirrhotic patients. Shock, Vol. $\left({ }^{0}\right)^{r}$,, PP. $\leqslant 0 \cdot-\leqslant \leqslant 0$ ,2011.

[11]R.de Franchis, A.Dell'Era. Non-invasive diagnosis of cirrhosis and the natural history of its complications. Best Practice \& Research Clinical Gastroenterology, Vol.21(1), PP.3-18,2007.

[12]P.Ginès, R.W.Schrier. Renal failure in cirrhosis. New England Journal of Medicine, Vol.361(13), PP.1279-1290,2009.

[13] T.Hassanein, F.Tofteng, R.Brown. MELD and SOFA scores as a predictor of transplant free survival time in patients with end stage liver disease complicated by intractable hepatic encephalopathy grades 3 and 4. Paper presented at the Gastroenterology,2005.

[14] M.Sheehan, B.Zeiler, S.Futterer. MELD scores, encephalopathy and survival in acute-on-chronic liver failure. Paper presented at the Gastroenterology,2005.

[15]F.Shafiq, A.Ijaz, M.Kashif. Chronic liver disease related admissions and mortality pattern in medical ICU. Pak J Gastroenterol, Vol.20, PP.72-74,2006.

[16] S.Shaikh, H.Ghani, S.Memon. MELD era: is this time to replace the original Child-Pugh score in patients with decompensated cirrhosis of liver. J
Coll Physicians Surg Pak, Vol.20(7), PP.432435,2010.

[17] B.De Jonghe, C.Cheval, B.Misset .Relationship between blood lactate and early hepatic dysfunction in acute circulatory failure. Journal of critical care, 14(1), 7-11, 1999.

[18] C.Y.Cheng, C.T.Kung, K.H.Wu. Liver cirrhosis affects serum lactate level measurement while assessing disease severity in patients with sepsis.2020,

[19]X.Zhu, Y.Zhang, Y.Nie. Serum lactate level predicts 6-months mortality in hospitalized patients with decompensated cirrhosis, 2019.

[20]A.Tas, E.Akbal, Y.Beyazit. Serum lactate level predict mortality in elderly patients with cirrhosis . Wiener klinische Wochenschrift, Vol.124(15-16), PP.520-525,2012.

[21] S.A.Sterling, M.A. Puskarich, A.E.Jones. The effect of liver disease on lactate normalization in severe sepsis and septic shock: a cohort study. Clinical and experimental emergency medicine, Vol.2(4), PP.197,2015.

[22] V.Das, P.Y.Boelle, A.Galbois. Cirrhotic patients in the medical intensive care unit: early prognosis and long-term survival. Critical Care Medicine, Vol.38(11), PP.2108-2116,2010.

[23] D.M.Heuman, S.G.Abou-Assi, A.Habib. Persistent ascites and low serum sodium identify patients with cirrhosis and low MELD scores who are at high risk for early death. Hepatology, Vol.40(4), PP.802-810,2004. 